\title{
Computer Network Fault Diagnosis Based On Neural Network
}

\author{
Wang Qian \\ Zibo Vocational Institute, Zibo, China, 255314 \\ $55976631 @ Q q . C o m$
}

\begin{abstract}
Computer network is one of the world's most important infrastructures in twenty-first Century, network fault diagnosis has become the focus of attention. With the development of artificial intelligence, using the neural network technology into the network fault diagnosis area can play an important role to the advantages of neural network in fault diagnosis. In this paper, the method is widely used, which is combined the self organizing feature map (SOM) neural network and multilayer feedforward neural network (BP): The result of the training samples using SOM neural network clustering algorithm is added to the original training samples and set a certain weight, through iterative update to the weight, in order to improve the convergence the speed of BP neural network. Using computer network fault diagnosis as a practical example for the computer simulation and analysis developes a set of computer network diagnosis system can provide reference and assistance for the work of theory research and application.
\end{abstract}

Keywords: Computer Network, Fault Diagnosis, Back-Propagation, Neural Networks

\section{Introduction}

Computer and continuous development of network technology and application, causing people to computer system security problem is getting much attention, because the computer system once destroyed, will cause significant economic losses using the unit, and seriously affect the normal work and the smooth development. Therefore, to enhance the security of computer systems and maintenance work, is one of the important tasks of information technology work. To some extent, network management is a term can have multiple interpretations. It may be operating on a small network of amateur administrators occasionally performed, or large communications provider network operations, companies and organizations staff at all times conducted.

Network fault diagnosis is the goal of the available time and maximize the network, improve the utilization rate of network equipment, network performance, service quality and safety, simplifying the mixed management under the network environment and reduce network operation cost control, to extend the service life of the network. The essence of fault diagnosis is pattern recognition, because of the diversity and complexity of network equipment and fault form, between the network fault symptom and fault condition is not a simple one one correspondence from the fault symptoms set to fault state set is a complex nonlinear mapping. Fault diagnosis is to detect the state information generated operating process of network equipment, extract the sign reflecting the network running status of equipment features from the detected signals, and to identify the state of equipment according to the symptoms and other diagnostic information, find the fault part, find the causes to find fault, 
puts forward the corresponding measures of troubleshooting. To complete the fault diagnosis.

Fault diagnosis is a new emerging technology on equipment operation, maintenance, began in 60 to 70 years of aviation, aerospace and nuclear engineering, it refers to a certain working environment lead to the identification of the nature and causes of the mechanical system a dysfunctional, judge the deterioration state occurring parts, and predict the development trend of the state of deterioration. The purpose of fault diagnosis is: the first is to improve the reliability of equipment operation, to avoid the equipment for the sudden failure and catastrophic damage caused enormous economic losses. The second is the accurate prediction of the running state of the equipment, ensure the maximize the function of the equipment and production efficiency. Third is to provide the technical foundation for the equipment repair system reform, save repair cost and human, material resources. In addition, the technology of fault diagnosis but also for the machine design, manufacture and operation of testing service.

The purpose of troubleshooting is to determine the equipment in operation inside the development and transfer trend implied fault, failure to identify dominant and dominant fault, you also need to assess the current state of the device, and make long-term forecasts of its state of deterioration trend. Process fault diagnosis can usually be summed up as signal acquisition, extraction and state identification signs three stages.

In the past ten years, with the cross-cutting and penetration mechanical vibration, friction science, equipment management, maintenance, reliability engineering, safety engineering, mechanics, intelligence and other related scientific frontier, making fault areas academic thinking has become increasingly active, attracted many experts in the field to participate in and promote the renovation of traditional disciplines and multi-door form a new growth point. From the method to fault diagnosis technology means there are a lot of development, a large number of scientific and technological achievements related to the emergence, development and penetration, also contributed to its development. Meanwhile, due to the rapid development of computer technology, more so fault diagnosis technology with unprecedented value and reputability. However, the penetration of each discipline, only change its ways and means, its fundamental purpose is still to obtain and interpret equipment operating status information, thus ensuring the normal operation of equipment and maintenance, as appropriate, to reduce or eliminate accidents. In all walks of life, due to the application fault diagnosis technology, not only effectively prevent the occurrence of many major accidents, but also made great economic and social benefits.

Fault diagnosis technology development has gone through three stages: the first stage is relatively simple due to equipment failure diagnosis relies on sensory organs expert or maintenance personnel, personal experience and can handle simple instrument fault diagnosis and troubleshooting work [1]. Sensor technology, the development of dynamic testing techniques and signal analysis technology makes diagnostic technology into the second stage, and has been widely used in the maintenance and reliability engineering. The early 1980s, due to the increasing complexity of machinery and equipment, intelligence and electron-mechanical integration, traditional diagnostic techniques have been unable to meet with the 
development of computer technology, artificial intelligence, expert systems technology, especially diagnostic technology into the third stage of development intelligent stage.

Intelligent Fault Diagnosis is based on the fault signal detection and processing, combined with expert knowledge in the field of artificial intelligence techniques and diagnostic reasoning, having given environment to the diagnosis object under the state's ability to identify and state prediction [2]. It applies to simulate human thinking process, diagnose problems need to solve complex logical reasoning, you can search for necessary diagnostic procedures and the use of expert knowledge and experience in the field to reach diagnostic purposes.

Intelligent Fault Diagnosis techniques include fuzzy technology, gray theory, pattern recognition, fault tree analysis, diagnostic expert systems. Just before the four technologies in a way to use the logic of knowledge, a partial solution to the diagnostic process such as information vague, incomplete, fault classification and location and other problems, and diagnosis expert system can in itself as a platform, integrated with other diagnostic technology, a hybrid intelligent fault diagnosis system. Intelligent diagnostic technology in general it refers narrowly expert systems.

Due to the inherent superior neural network adaptation and learning ability, so that in many areas has been widely applied to solve many difficult problems of traditional methods. Nonlinear adaptive neural network-specific information processing capability to overcome the traditional artificial intelligence methods for intuition, such as mode, voice recognition, unstructured information processing deficiencies, so that in the nervous expert systems, pattern recognition, intelligent control, the combination of optimization, forecasting and other fields has been successfully applied [3]. Neural network with other traditional methods combined will promote artificial intelligence and information processing technology continues to develop.

In this study, fault recognition neural network applications in computer-based network made some attempts, expect the study of computer network failure, the use of the characteristics of neural networks provide the basis for computer network diagnostics, to computer network optimization purposes.

\section{Related Works}

AI (Artificial Intelligence) is proposed in 1956 by j. Mc Carthy and others in the academic discussion about the machine simulation Intelligence put forward at the meeting. Artificial Intelligence (AI) is the study of machine intelligence and intelligent machines of high and new technology subject, is to simulate, extend and expand people's intelligence, to realize the automation of some mental work technical foundation, is an important scientific way to explore the mysteries of the human brain and computer application in the wide areas.

So far, theoretical research and application of artificial intelligence has achieved many important results, the main application of artificial intelligence in the following three aspects:

(1) machine thinking and thinking machines. Such as: heuristic procedures, expert systems, knowledge engineering, machine learning, proving, intelligent computer, 
machine learning, inference engine, game machines, neural cell model, artificial neural networks.

(2) machine perception and perception machine. Such as: text, images, objects King, sounds, pattern recognition, haptic machine, smart sensors.

(3) machine behaves machine. Such as: adaptive, self-stabilization, self-seeking superior intelligent control, management, decision-making behavior, the robot behavior.

Today, although artificial intelligence has been widely used, but according to the traditional views and methods, it is impossible to achieve a major breakthrough in the near future [4]. This is because the AI is facing difficulties as follows:

(1) distribution of human thinking and parallelism, randomness, uncertainty, determines the use of the machine type von Neumann architecture to simulate intelligent human behavior is not rational.

(2) existing brain models and mechanisms can not exactly reflect the actual mechanism of the human mind.

(3) human thinking mode and operation mechanism should society organizations there

is a certain similarity, now research in this area is still very small.

(4) mechanism of the human mind could not draw enough nutrients in the natural development (especially biological evolution) in.

(5)Each school AI cross between the perspectives and methods, the degree of penetration and comprehensive enough.

(6) AI using an independent problem-solving model for a single, does not reflect the intrinsic link of the human mind and the nature of social activities.

(7)intelligence issues often include a huge amount of parallelism and calculation must aims to provide high-performance computing resources to adapt to the environment (including the machine performance, communications, computing mode, the system structure).

(8) solve large problems when intelligence resources to provide transparency for the programmer is not enough.

AI development for the difficulties faced and the actual needs of large-scale intelligent problem solving $[5,6]$, research appeared to intersect with the penetration of a variety of disciplines, AI and computer networks combined with the establishment of the human mind to adapt to distributed parallel processing model, structure suitable AI research and test platform is the new trend. Throughout AI research in recent years, the current AI research focuses are:

(1) Knowledge Representation and Reasoning: WEB major research-oriented knowledge representation and reasoning as well as search and constraint issues. The new mechanism for non-precise reasoning - and probabilistic reasoning and knowledge representation, combined with learning mechanism, will become a hot research topic in the coming years.

(2) Distributed Artificial Intelligence (DAI) and multi-agent systems (MAS).

(3) Machine learning and data mining.

(4) Research neural networks and genetic algorithms.

1943 and mathematical logician W.Pitts psychologist W.McCutloch first proposed a simple neural network model (referred to as MP model), the date, there have been dozens of major network structure and a variety of network learning algorithm, see 
Ref. [21-23]. One of the most representative are: MP model (McCulloch and Pitts, 1943), Hebb rule (Hebb, 1949), Percepton Perceptron (Rosenblatt, 1957), Hopfield network (Hopfield, 1984), BP network (Rumelhart, 1985), Boltzmann machine (Hinton, 1984), dynamic BP neural network (Werbos, 1990), as well as fuzzy neural network [7]. Corresponding learning algorithms include: standard BP algorithm multilayer feedforward neural network and its various modifications, adaptive variable step learning algorithm, least squares learning algorithm, second-order learning algorithm, high-end fast learning algorithm, genetic algorithm dynamic programming learning algorithms, simulated annealing, and fuzzy neural network learning algorithm further incentive gradient learning algorithm.

Have been in fault diagnosis pattern recognition methods are:

(1) statistical classification methods. This method is the use of the distribution pattern and the like, i.e., the direct use of various types of probability density functions, the posterior probability, or implicitly by the above concept of classification. According to the statistical criterion to divide classification methods, including minimum standards and minimum loss probability misjudgment judgment rule.

(2) clustering classification. To avoid the difficulty of estimating the probability density, under certain conditions, according to the similarity of the sample set sample space is divided into several subsets, indicating the quality of the clustering criterion function is maximized.

(3) fuzzy pattern recognition. This method is the use of the theory and methods of fuzzy mathematics to solve the problem of pattern recognition, the recognition results are applicable to the classification requirements of the object or fuzziness of the occasion. Currently, there are a lot of fuzzy pattern recognition method, the simplest and most common is the largest membership principle.

Aiming at the complex nonlinear mapping relationship between faults and symptoms, Jin Lin and Zhang hong proposed a new diagnostic system architecture models. The model uses fuzzy neural network diagnostic matrix obtained from the statistical previous diagnosis instance, transform fuzzy inference, through dynamic weights Comprehensive Comprehensive inference rules to draw final conclusions of the diagnosis, thus solving the problem of non-orthogonal rules, so that the diagnosis result is statistically significant, and the conventional method to solve the difficulties to obtain rules and, in use, a problem is not easy to modify. Shi Yongsheng and Song Yunxue overcomed the shortcoming of the single application of BP algorithm, using genetic algorithm (GA) to improve it, to establish a diagnosis model based on genetic algorithms and the combination of BP neural network [8]. Wang Zhengwu and Zhang Ruiping worked on the feasibility of neural network for fault diagnosis, the basic principles of the diagnostic process analysis, and parameter optimization process for a more in-depth research and draw an ideal diagnostic results.. Ping Jingang, Li Hang propose a neural network method for remote diagnosis and remote network fault diagnosis model based on this method to achieve a diagnosis of intelligence, and finally through an experiment to test the feasibility of this model. Zhu Xianglin, Jinyan Cai, He Qiang proposed implementation process based on self-organizing neural network troubleshooting platform interface module. Classification of samples with Delphi entry work and platform graphical user interface features, and the samples were relatively simple cluster analysis. 
Distributed network fault diagnosis model based on Bayes classifier proposed a distributed network fault diagnosis system model framework based Agent, the model for Bayesian classification theory of promotion. Model prior knowledge and observation data together, which greatly improved the diagnostic performance of the system. Model uses a certain status check and verification strategies to ensure their own safety and the safety of Agent Communication provides a common framework for network fault diagnosis system. Qi Chung proposed network diagnostic system based on BP neural network. BP neural network fault diagnosis system for research and design, and through simulation examples, there is room for research. Li Qian-mu high redundancy for traditional fault Knowledge difficult co-existence and stability defects RSFBP proposed algorithm, the integrated use of neural networks and rough set theory, to achieve the learning sample purification [9]. The experimental results show that this method achieved during network fault diagnosis system can achieve better results. Based on the above results of existing neural network in fault diagnosis, the paper from the use of SOM neural network and BP neural network combined with the start, in the neural network algorithm to make further improvements to the computer network failure to make examples and simulation the work.

\section{Proposed Scheme}

\subsection{SOM Neural Network Model}

Self-organizing feature map SOM (Self-Organizing Feature Map) neural network is put forward by the Finnish scholar Teuvo Kohonen. The network is an unsupervised by an array of fully connected neurons composed of self-organizing, self-learning network.

Multilayer Perceptron learning and classification are known a priori knowledge of certain conditions, namely, the right to adjust the value of the network is carried out under the supervision of the case. In practical applications, sometimes does not provide the required prior knowledge, which requires the network has the ability to be able to self-learning. Kohonen self-organizing feature map is this proposed neural network has self-learning function. This network is based on the results of scientific studies of brain physiology and raised. Scientific research shows that the brain: neurons are arranged according to a certain feeling of being passed laws in an orderly manner, and this arrangement is often felt by external stimuli reflect certain physical characteristics. For example, in the auditory system, the nerve cells and fibers in accordance with their frequency distribution of the most sensitive arrangement. Kohonen believes that the neural network in an external input, will be divided into different regions, different areas for different models with different response characteristics, that different neurons respond best to the different nature of the excitation signal, thereby forming a ordered Figure topological sense, also called feature map, is actually a nonlinear relationship, it will signal space topology of each mode is almost invariably reflected in the output response of each neuron ${ }^{[10]}$. The map is by unsupervised adaptive process is completed, it is also called self-organizing feature map.

SOM network learning algorithm process is as follows: 
(1) initialization. On $\mathbf{N}$ input neurons with connection weights from the output neurons to the output neurons are assigned smaller weights.

(2) provide new input pattern $X$.

(3) calculate the distance $d_{j}$, namely the distance is between each input sample and each output neuron $\mathrm{j}$ :

$$
d_{j}=\left\|X-W_{j}\right\|=\sqrt{\sum_{i=1}^{N}\left[x_{i}(t)-w_{i j}(t)\right]^{2}}
$$

And find the very neuron having the smallest distance $j^{*}$, namely determine a unit $\mathrm{k}$, such that for any $\mathrm{j}$ makes $d_{k}=\min \left(d_{j}\right)$.

(4) shows a surrounding neighborhood $s_{k}(t)$.

(5) corrected output neurons i' "neighboring neurons "weights:

$$
w_{i j}(t+1)=w_{i j}(t)+\eta(t)\left[x_{i}(t)-w_{i j}(t)\right]
$$

Among them, $\eta$ is Gain entry and will be zero with time gradually decreased. General value for

(6) calculate the output $o_{k}$ :

$$
w_{i j}(t+1)=w_{i j}(t)+\eta(t)\left[x_{i}(t)-w_{i j}(t)\right]
$$

$$
O_{k}=f\left(\min \left\|X-W_{j}\right\|\right)
$$

Generally speaking, $f(\square)$ is typically $0-1$ functions or other non-linear function (7) provide new learning sample repeat the learning process.

\subsection{Application of Neural Network Fault Diagnosis}

As an adaptive neural network pattern recognition technology, does not need to be given in advance of knowledge and experience related to the discriminant function mode, it automatically required to form a decision-making region through its own learning mechanisms [11]. Characteristics of the network by its topology properties of neurons, learning and training rules of the decision. It can make full use of the status information, the status information from each of the different training to obtain certain mappings. And the network can continue the learning, if the environment changes, the mapping may also be adjusted adaptively.

For fault diagnosis based on neural network, the input node corresponds to a network fault symptoms, output node corresponds to the cause of the failure. First, using a set of fault samples to train the network to determine (the transfer function of the number of intermediate layers and neurons) and the structure of the network parameters (connections between neurons weights and thresholds) [12]. After the completion of network training, the classification is based on the failure mode of a given set of signs, to achieve symptom set to a non-linear mapping between failures, having the following characteristics:

(1) unknown or system model can be used to model more complex systems, and fault pattern recognition of non-linear systems.

(2) both fault signal pattern transform and feature extraction. 
(3) on the system contain uncertainties, are less sensitive to noise and input mode incomplete situation.

(4) can be used for troubleshooting complex multi-mode.

(5) can be used for off-line diagnostics, but also to adapt to the real-time monitoring requirements.

Diagnostic process based on neural network is divided into two steps:

(1) based on a certain number of training samples for training the neural network to obtain diagnostic results desired.

(2) According to the input of the current diagnostic system for diagnosis, diagnosis is the use of the procedure before the neural network computing. Before learning and diagnosis usually requires the raw data for diagnosis and appropriate training sample data processing, including pre-processing and feature selection, extraction. The purpose is to provide appropriate training samples for diagnostic inputs and diagnose network.

Although neural networks and troubleshooting traditional two different diagnostic methods, but the two are closely linked. A data processing method based on wavelet analysis can provide a feature vector can be used for neural network diagnosis.

Forward learning algorithm BP and RBF networks belong teachers type. This algorithm has a good ability to promote the model, the effect for fault pattern recognition better. Trained BP network and RBF network has a certain density, ergodicity and compatibility.

The general steps for troubleshooting network using BP as follows:

(1) determine a reasonable network structure and model, especially the choice of the number of neurons in the middle layer of the network is to identify key network structure and network performance.

(2) determine the training set and test set. Training sample set is used to train the network, while the test set is used to monitor the effectiveness of training and the promotion of network capacity. In general, the training sample set should not only cover all the failure modes class comprehensive data, there should be some representation, but must also ensure the effectiveness of learning.

(3) according to the training sample set to train the network, the test results shall be trained neural network fault diagnosis knowledge base.

ART network and the networks are self-organizing network SOM unsupervised competitive learning. ART network online learning. To the network to provide a set of samples, the network automatically generates a set of classification models. SOM learning using offline mode, can well feature extraction, suitable for use as the most neighbor classifier. Although the performance of these models is not the promotion of BP and RBF Network is good, but the self-organization and adaptive features which have applications in complex systems for fault diagnosis have a good theoretical basis.

\subsection{LM algorithm introduced}

Strategies to improve the convergence rate of the BP algorithm is divided into two main categories: heuristic techniques and numerical optimization techniques. The former involves the use of an adaptive learning rate and adding momentum, corresponding to the MATLAB Traingdx algorithm; the latter include conjugate gradient algorithm and LM algorithm. In recent years, there are some other improvement strategies, such as the BP algorithm and fuzzy logic or genetic algorithms and other technologies. 
Let $x_{k}$ be the first step iterative approximation $\mathrm{k}, \mathrm{F}$ is the objective function.

$$
F(x)=\sum_{i=1}^{N} V_{i}^{2}(x)=v^{T}(x) v(x)
$$

In the above formula,$v(x)=\left(v_{1}(x), v_{2}(x), \ldots, v_{N}(x)\right)^{T}$. Then the LM algorithm is as follows:

$$
\Delta x_{k}=x_{k-1}-x_{k}=-\left[H\left(x_{k}\right)+\mu_{K} I\right]^{-1} J^{T}\left(x_{k}\right) v\left(x_{k}\right)
$$

In the above formula, $\mathrm{J}$ is the Jacobi matrix of $\mathrm{F}$.

$$
J(x)=\left[\begin{array}{cccc}
\frac{\partial v_{1}(x)}{\partial x_{1}} & \frac{\partial v_{1}(x)}{\partial x_{2}} & \ldots & \frac{\partial v_{1}(x)}{\partial x_{n}} \\
\frac{\partial v_{2}(x)}{\partial x_{1}} & \frac{\partial v_{2}(x)}{\partial x_{2}} & \ldots & \frac{\partial v_{2}(x)}{\partial x_{n}} \\
\vdots & \vdots & \vdots & \vdots \\
\frac{\partial v_{N}(x)}{\partial x_{1}} & \frac{\partial v_{N}(x)}{\partial x_{2}} & \cdots & \frac{\partial v_{N}(x)}{\partial x_{n}}
\end{array}\right]
$$

$\mathrm{H}$ is an approximation of the Hesse matrix of the matrix $\mathrm{F}$, taken as:

$$
H\left(x_{k}\right)=J^{T}\left(x_{k}\right) J\left(x_{k}\right)
$$

$\mu_{\kappa}$ is a parameter greater than zero LM algorithm used internally for control of LM algorithm iterations. When $\mu_{\kappa}$ is close to zero, LM algorithm is close to the Gauss-Newton method. When $\mu_{\kappa}$ is large, LM algorithm approximates the steepest descent method.

\section{Experimental Results and Analysis}

According to the method described earlier in this section using the previous method combines the idea, the first of the training sample data SOM neural network clustering, and then set the weight on the output, then the BP neural network model, using the updated sample data on nerve network training, the use of parallel algorithms on LM algorithm improved training algorithm.
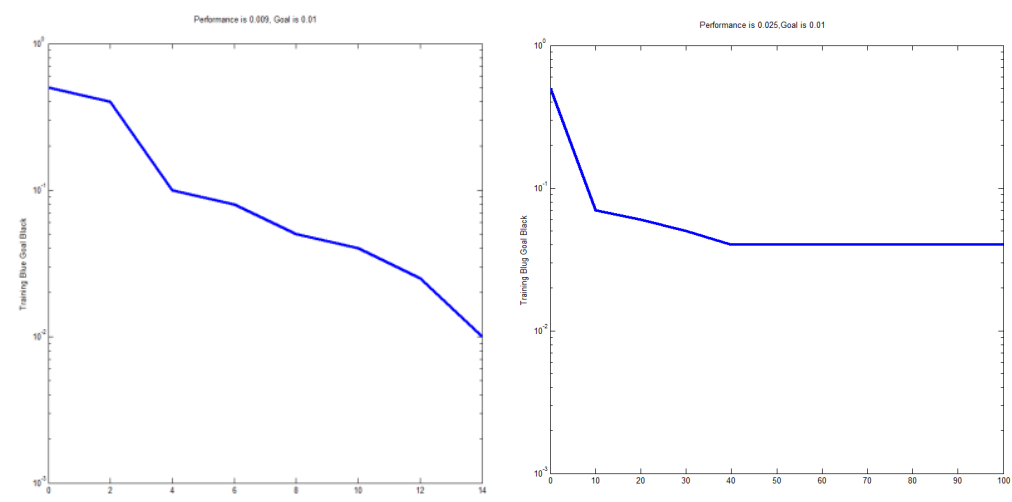

Figure 1 and Figure 2. Comparison of Combined Algorithm with the Original Algorithm of the Training Process 
Figures 1 and 2 can be seen by using the SOM method and LM method more general method of combining BP neural network has faster convergence.

Table 1 shows the average BP neural network and BP algorithm using simulation results for the same sample conducted.

Table 1. Result of Emulation

\begin{tabular}{|l|l|l|l|}
\hline B1 & B2 & B3 & B4 \\
\hline 0.0080 & 0.0765 & 0.0014 & 0.9999 \\
\hline 0.8674 & 0.0000 & 0.0000 & 0.0000 \\
\hline 0.0000 & 0.8521 & 0.0006 & 0.0000 \\
\hline 0.0008 & 0.0000 & 0.9540 & 0.1775 \\
\hline 0.7859 & 0.1548 & 0.0025 & 0.0541 \\
\hline 0.0155 & 0.9254 & 0.0052 & 0.0845 \\
\hline 0.9145 & 0.0005 & 0.1231 & 0.0089 \\
\hline 0.0754 & 0.0063 & 0.8547 & 0.0015 \\
\hline 0.0012 & 0.9005 & 0.2145 & 0.0036 \\
\hline 0.0001 & 0.0231 & 0.9254 & 0.0003 \\
\hline 0.9958 & 0.0001 & 0.0047 & 0.0006 \\
\hline 0.0003 & 0.9914 & 0.1587 & 0.0256 \\
\hline
\end{tabular}

SOM method using visible and LM method more general method of combining BP neural network has a higher simulation accuracy. Thus the use of SOM methods and LM incorporated methods to improve the accuracy of simulation sample has a good effect. Visible make SOM methods and LM method combines not only improves accuracy, and greatly reduce the training times.

\section{Conclusion}

In this paper, a computer network fault diagnosis were studied using the method of SOM methods and LM computer network fault simulation and emulation. SOM neural network based on self-organization of teachers belonging to non-competitive learning network for computer network fault diagnosis without pre-training samples indicate the type of fault actually belongs, have a good ability to clustering. Use way to add weight effectively combine SOM neural network and BP neural network up and LM algorithm using parallel algorithms made some improvements. There is a certain sense diagnosis showed by example.

\section{References}

[1] Z. Pawlak, "Rough Set Theory and its Applications to Data Analysis", Cybernetics \& Systems, vol. 29, no. 7, (1998), pp. 661-688.

[2] T. S. Bi, Y. X. Ni and C. M. Shen, "A Novel ANN Fault Diagnosis System for Power Systems Using Dual GA Loops In ANN Training Power Engineering Society Summer Meeting”, IEEE, vol. 1, (2000), pp. $425-430$.

[3] R. Saeks, M. Lothers and R. Pap, "A Neural Network Based Approach to Fault Diagnosis in Aerospace Systems”, AUTOTESTCON'92, IEEE Systems Readiness Technology Conference, Conference Record. IEEE, (1992), pp. 271-273. 
[4] A. A. El-Fergany, M. T. Yousef and A. A. El-Alaily, "Fault Diagnosis in Power Systems-Substation Level-Through Hybrid Artificial Neural Networks And Expert System", Transmission And Distribution Conference And Exposition, 2001 IEEE/PES. IEEE, vol. 1, (2001), pp. 207-211.

[5] F. Lv, H. L. Du and J. H. Yang, "The Electric Actuator's Fault Diagnosis Based on Information Fusion[C]//Machine Learning and Cybernetics”, 2007 International Conference on. IEEE, vol. 2, (2007), pp. 1055-1060.

[6] Z. Wen and H. Su, "Intelligent Implementation Technologies on Sensing Dam Safety Based on Neural Network", Power and Energy Engineering Conference, 2009. APPEEC 2009. Asia-Pacific, IEEE, (2009), pp. 1-4.

[7] T. Yang, "A Method Of Neuro-Fuzzy Computing For Effective Fault Diagnosis", Control Applications, 2005, CCA 2005, Proceedings of 2005 IEEE Conference on. IEEE, (2005), pp. 256-259.

[8] J. Yuqin, W. Peixia and L. Yue, "Study Of Manufacturing System Based On Neural Network Multi-Sensor Data Fusion And Its Application”, Robotics, Intelligent Systems and Signal Processing, 2003. Proceedings. 2003 IEEE International Conference on. IEEE, vol. 2, (2003), pp. 1022-1026.

[9] A. Barua, P. Kabisatpathy and S. Sinha, "A Method To Diagnose Faults In Analog Integrated Circuits Using Artificial Neural Networks With Pseudorandom Noise As Stimulus", Electronics, Circuits and Systems, 2003, ICECS 2003, Proceedings of the 2003 10th IEEE International Conference, IEEE, vol. 1, (2003), pp. 356-359.

[10] P. Q. Dzung, P. Q. Vinh and N. Van Nho, "The Development of Artificial Neural Network Space Vector PWM and Diagnostic Controller for Voltage Source Inverter", Power India Conference, 2006 IEEE, pp. 8.

[11] H. H. Lee, P. Q. Dzung and T. P. Hoa, "The Investigation Of ANN Space Vector PWM And Diagnostic Controller For Four Switch Three Phase Inverter Fed Induction Motor Drive", Industrial Technology, 2009, ICIT 2009, IEEE International Conference on IEEE, (2009), pp. 1-6.

[12] C. Zhang and D. Cai, "Application of Neural Network to Faults Diagnosis of Nonlinear Circuits", Computer Science and Network Technology (ICCSNT), 2011 International Conference on. IEEE, vol. 3, (2011), pp. 1932-1935.

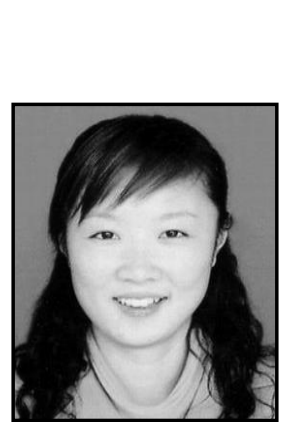

\section{Author}

Wang Qian received the B.S. degree in Computer science and technology from Huanggang Normal University and the MPA degree of Software engineering from University of Electronic Science and technology of China in 2003 and 2010 respectively. She is lecturer at Zibo vocational institute where she is backbone teacher of software department. She is currently researching on algorithm design, Information and software project development, Network engineering. 
International Journal of Future Generation Communication and Networking Vol. 8, No. 5 (2015) 